\title{
BMJ Open Evaluation of metformin in combination with rifampicin containing antituberculosis therapy in patients with new, smear-positive pulmonary tuberculosis (METRIF): study protocol for a randomised clinical trial
}

\author{
Chandrasekaran Padmapriyadarsini, ${ }^{1}$ Perumal K Bhavani, ${ }^{1}$ Mohan Natrajan, ${ }^{1}$ \\ Chinnayan Ponnuraja, ${ }^{1}$ Hemanth Kumar, ${ }^{1}$ Sivaramakrishnan N Gomathy, ${ }^{1}$ \\ Randeep Guleria, ${ }^{2}$ Shaheed M Jawahar, ${ }^{3}$ Manjula Singh, ${ }^{4}$ Tanjore Balganesh, ${ }^{3}$ \\ Soumya Swaminathan ${ }^{4,5}$
}

To cite: Padmapriyadarsini C, Bhavani PK, Natrajan M, et al. Evaluation of metformin in combination with rifampicin containing antituberculosis therapy in patients with new, smear-positive pulmonary tuberculosis (METRIF): study protocol for a randomised clinical trial. BMJ Open 2019;9:e024363. doi:10.1136/ bmjopen-2018-024363

- Prepublication history for this paper is available online. To view these files, please visit the journal online (http://dx.doi. org/10.1136/bmjopen-2018024363).

Received 1 June 2018 Revised 21 November 2018 Accepted 29 November 2018

D) Check for updates

(c) Author(s) (or their employer(s)) 2019. Re-use permitted under CC BY-NC. No commercial re-use. See rights and permissions. Published by BMJ.

For numbered affiliations see end of article.

Correspondence to Dr Chandrasekaran Padmapriyadarsini; pcorchids@gmail.com

\section{ABSTRACT}

Introduction Shorter duration of treatment for the management of drug-susceptible pulmonary tuberculosis (TB) would be a significant improvement in the care of patients suffering from the disease. Besides newer drugs and regimens, other modalities like host-directed therapy are also being suggested to reach this goal. This study's objective is to assess the efficacy and safety of metformincontaining anti-TB treatment (ATT) regimen in comparison to the standard 6-month ATT regimen in the treatment of patients with newly diagnosed sputum smear-positive drug-sensitive pulmonary TB.

Methods and analysis We are conducting a multicentric, randomised open-label controlled clinical trial to achieve the study objective. The intervention group will receive isoniazid $(H)$, rifampicin $(\mathrm{R})$, ethambutol $(\mathrm{E})$ and pyrazinamide (Z) along with $1000 \mathrm{mg}$ of daily metformin (Met) for the first 2 months while the control group will receive only HRZE. After 2 months, both the groups will receive HRE daily for 4 months. The primary endpoint is time to sputum culture conversion. Secondary endpoints will include time to detection of Mycobacterium tuberculosis in sputum, pharmacokinetics and pharmacogenomics of study drugs, drug-drug interactions, safety and tolerability of the various combinations and measurement of autophagy and immune responses in the study participants.

Ethics and dissemination The ethics committee of the participating institutes have approved the study. Results from this trial will contribute to evidence towards constructing a shorter, effective and safe regimen for patients with TB. The results will be shared widely with the National Programme managers, policymakers and stakeholders through open access publications, dissemination meetings, conference abstracts and policy briefs. This is expected to provide a new standard of care for drug-sensitive patients with pulmonary TB who will not only reduce the number of clinic visits and lost to followup of patients from treatment but also reduce the burden on the healthcare system.
Strengths and limitations of this study

- Study design, randomised controlled clinical trial, will add considerable power to the study results and conclusion by decreasing bias (selection bias and observer bias) and minimising confounding of unequal distribution.

- This study design of randomised controlled clinical trial with stratification will also control group allocation, enhancing similarity of baseline features including disease severity in the two study arms. This will help us in subgroup analysis improving usefulness for clinical practice.

- Due to the strict exclusion and inclusion criteria of the clinical trial, the results from the clinical trial may not be generalisable to all groups of patients with tuberculosis.

- Due to the multicentric nature of the study, the heterogeneity of data may be present.

- Relapse rates may not be known in this trial as the study duration is only for 6 months of treatment as per the study objective.

Trial registration number CTRI/2018/01/011176; Preresults.

\section{INTRODUCTION}

Globally in 2016, there were an estimated 10.4 million new cases of tuberculosis (TB) with five countries, India, Indonesia, China, Philippines and Pakistan, accounting for $56 \%$ of the total cases. ${ }^{1}$ There were an estimated 1.3 million TB deaths in 2016 among HIV-negative people and an additional 374000 deaths among people living with HIV. ${ }^{1}$ Though effective regimens are available for the treatment 
of drug-sensitive TB with more than 95\% cure, the long duration of such regimens has posed problems for TB treatment and control. This, along with drug toxicity, results in poor adherence to treatment resulting in the emergence of drug resistance.

All these have led to an urgent need for more efficient anti-TB drugs, regimens as well as for newer modalities of treating TB. Drugs targeting the TB bacilli can result in the emergence of drug tolerance and resistance, thereby worsening the overall treatment outcomes. Thus, there exists a need to consider alternate modalities such as enhancing the host immune system for a faster and complete elimination of the TB bacilli. ${ }^{23}$ An efficient and functional immune system is essential to restrain and curb the growth of TB bacilli in the host. Yet, the TB bacilli can still elude the host immune responses, infect the host cells and either multiply or maintain long-term latency in those cells. ${ }^{45}$ 'Host-targeted' adjunct therapeutic strategies not only augment protective host immune responses but also reduce the chance of development of microbial resistance.

One of the host cell innate antimicrobial arsenals includes the capacity to destroy intracellular pathogens using the phagosomal machinery or autophagy pathway. Intracellular pathogens are effectively controlled by autophagy that is regulated by adenosine monophosphate-activated protein kinase (AMPK) ${ }^{67}$ The Mycobacterium tuberculosis virulence results from perturbations in the autophagy network and AMPK signalling. ${ }^{8}$ The antidiabetic drug metformin (MET; 1, 1-dimethyl biguanide) is an AMPK modulator that inhibits the intracellular growth of $M$. tuberculosis, restricts disease immunopathology and enhances the efficacy of conventional anti-TB drug. ${ }^{9}$ Given these promising findings, we plan to test whether the existing approved antidiabetic drug, metformin added to the existing anti-TB regimen, with its defined effects on host cell functions could be repurposed for effective and faster treatment of TB as compared with the current standard of care anti-TB regimens.

The primary objective of this study-the METRIF study-is to study the antibacterial activity, in terms of time to sputum culture conversion of metformin-containing anti-TB treatment (ATT) regimen instituted during the initial 8 weeks of treatment in patients with newly diagnosed sputum smear-positive pulmonary TB. Secondary objectives of the study are:

i. To compare the time to detection (TTD) of M. tuberculosis in culture in the group receiving metformin-containing regimen with the control group receiving ATT alone

ii. To study the autophagy-enhancing effect and host immune responses in the two groups

iii. To examine the postdosing serum concentration of anti-TB drugs and metformin, their interactions and the impact of genomics on these parameters (pharmacokinetics (PK) and pharmacogenomics) and iv. To evaluate the safety and tolerability of metformin by measuring the incidence of treatment-emergent adverse events.

\section{METHODS AND ANALYSIS}

\section{Study design and oversight}

METRIF is a multisite, randomised, open-labelled, parallel arm, controlled clinical trial comparing the time to sputum culture conversion among patients with pulmonary TB receiving ATT with metformin (experimental arm) compared with those receiving ATT alone (control arm). The study is randomising 316 participants to one of the two treatment arms in a 1:1 allocation. The study is sponsored by the India TB Research Consortium of the Indian Council of Medical Research and Open Source Pharma Foundation and implemented by the National Institute of Research in Tuberculosis (NIRT), together with other specialised institutes. The institutional ethics committee of NIRT has approved the study (NIRT-IEC ID: 2017030, dated 14 December 2017) and National AIDS Research Institute (NARI) (NARI EC/2018-10 dated 16 February 2018) and will begin enrollment tentatively by 15 June 2018.

\section{Study setting}

We will implement METRIF study at three sites in India - NIRT, Chennai and its satellite centres in Madurai and Vellore, All India Institute of Medical Sciences, New Delhi and NARI, Pune. These sites will recruit study participants from academic institutions/hospitals as well as community clinics.

\section{Study patients and eligibility}

Adult patients previously untreated and newly diagnosed with pulmonary TB with at least two sputum smear sample, collected on two different occasions, positive for acid-fast bacilli and susceptible to rifampicin detected by cartridge-based nucleic acid amplification test will be eligible for the study. Table 1 provides the detailed inclusion and exclusion criteria. Patients who meet these criteria at presentation and attending the identified study sites will be approached to participate in the study.

\section{Study regimen and drug dosing}

We will randomly assign eligible patients who have provided written informed consent to one of the study regimens in a ratio of $1: 1$. The site principal investigator or his/her nominee who is capable of answering all the trial-related questions from the participants will obtain the study consent. Study participation will last 6 months: during the first 2 months, participants will receive the randomly assigned regimen of either daily ATT with metformin or only ATT.

(1) Test regimen - metformin+isoniazid+rifampicin+pyrazinamide+ethambutol daily

[2MetHREZ] or

(2) Control regimen - isoniazid+rifampicin+pyrazinamide+ethambutol daily $\left[2 \mathrm{HRZE}_{7}\right]$ 
Table 1 Eligibility criteria

Inclusion criteria
Age 18 years and above
Is willing to undergo HIV testing
Has body weight between $30 \mathrm{~kg}$ and $65 \mathrm{~kg}$
Patient has never received treatment with multidrug anti-TB
therapy for more than a week.
Is willing to use an effective contraceptive method during the
study period

Is willing to attend a treatment centre for supervised treatment and remain within the study area limit

Is willing to sign the informed consent form and adhere to trial
procedures and follow-up

Consents for home visits by the study team

\section{Exclusion criteria}

Is pregnant or breastfeeding

Has extrapulmonary TB or drug-resistant TB

Has body weight $<30 \mathrm{~kg}$ or $>65 \mathrm{~kg}$

Has a prior history of exposure to anti-TB treatment for more than a week

Has a history of liver disease or current amino alanine transferase greater than three times the upper limit of normal (ULN) or total bilirubin concentration greater than 2.5 times the ULN

Is serology positive for hepatitis B virus surface antigen or hepatitis $\mathrm{C}$ virus antibody

Has concomitant psychiatric illness or seizures

Has concomitant diabetes mellitus or random blood sugar $>200 \mathrm{mg}$ or fasting blood sugar $>140 \mathrm{mg} / \mathrm{dL}$ Has serum creatinine $>1.2 \mathrm{mg} / \mathrm{dL}$ or blood urea $>43 \mathrm{mg} / \mathrm{dL}$
After 2 months, all study participants in both the arms will receive the standard 4-month continuation phase of HRE daily. Treatment will be supervised and directly observed at a health facility or by directly observed therapy (DOT) provider. Metformin will be dosed at $500 \mathrm{mg}$ once daily for the 1 st week and then $1000 \mathrm{mg}$ once daily for the remaining period of 7 weeks. Dosing of the other anti-TB drugs will be based on weight bands as shown in table 2 .

We will centrally procure all drugs (H, R, E, Z and Met) to be used in the trial, check for their stated content by validated methods using high-performance liquid chromatography at NIRT clinical pharmacology department, before dispatching it to the enrolling sites for administration to patients.

\section{Treatment allocation}

Permuted block randomisation will be done centrally using a computer-generated list of random numbers, stratified by presence or absence of cavities in chest X-ray and highest sputum smear grading at baseline $(<2$ or $>2)$. Separate randomisation lists for each combination of strata for each site will be prepared in advance by an independent statistician, using varying block sizes. Allocation codes will be generated at the central location and at the time of patient's admission to the study; the primary statistician through email will inform the site the regimen based on appropriate stratification factors.

\begin{tabular}{lllll}
\hline Table 2 & Weight-based dosing of anti-tuberculosis drugs \\
\hline $\begin{array}{l}\text { Weight } \\
\text { band }\end{array}$ & Isoniazid & Rifampicin & Pyrazinamide & Ethambutol \\
\hline $30-39 \mathrm{~kg}$ & $150 \mathrm{mg}$ & $300 \mathrm{mg}$ & $800 \mathrm{mg}$ & $550 \mathrm{mg}$ \\
$40-54 \mathrm{~kg}$ & $225 \mathrm{mg}$ & $450 \mathrm{mg}$ & $1200 \mathrm{mg}$ & $825 \mathrm{mg}$ \\
\hline $55-65 \mathrm{~kg}$ & $300 \mathrm{mg}$ & $600 \mathrm{mg}$ & $1600 \mathrm{mg}$ & $1100 \mathrm{mg}$ \\
\hline
\end{tabular}

\section{Recruitment process}

We will screen all patients with newly diagnosed sputum smear-positive pulmonary TB attending the chest clinics at the study sites for study eligibility. Table 3 details the various study procedures. At their first visit, the study will be explained, including the potential risks and benefits associated with participation. We will obtain informed written consent before any protocol-specific screening procedures are carried out. Consenting participants will undergo sputum testing for smear, culture (both solid and liquid media) and rifampicin resistance testing by GeneXpert. Blood samples will be obtained for HIV antibodies (unless the patient is already known to be HIV positive), liver and renal function tests and blood sugar levels. Patients will be reassessed for eligibility when returning with their investigation results. Those patients who do not have rifampicin resistance and are willing to take part in the study will sign an enrolment consent form (or a thumb print in the presence of a witness, if illiterate), and randomised to one of the study regimens. During the first 2 months of treatment, all patients will undergo weekly sputum testing for M. tuberculosis by smear, liquid and solid cultures and sparse pharmacokinetics of ATT drugs and metformin. A subset of patients will undergo intense pharmacokinetic study. Randomised patients have an additional blood investigation for immunological and autophagy biomarkers ( $\mathrm{T}$ cell, monocyte and dendritic cell functions both ex vivo and following stimulation with TB antigens including Purified Protein Derivative (PPD) and early secretory antigenic target, ESAT-6/CFP-10, Culture filtrate Protein, estimation of $\mathrm{C}$ reactive protein, tumour necrosis factor- $\alpha$ and other cytokines) pre and post metformin containing ATT. 


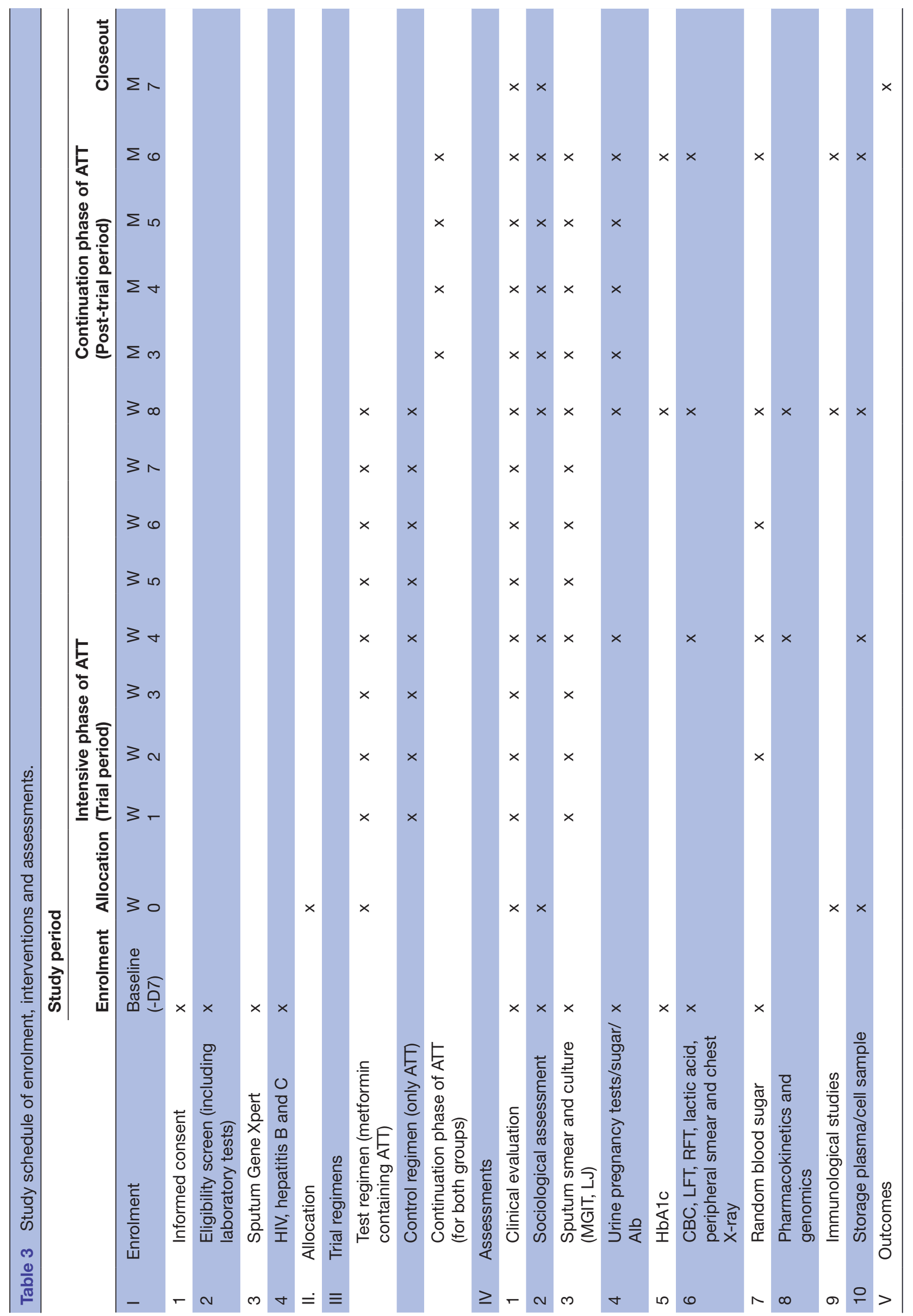

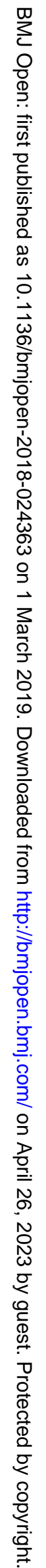


We will refer patients ineligible or unwilling to participate in the study to the national programme for treatment as per the existing guidelines.

\section{Treatment delivery, compliance and retention}

ATT will be administered under direct observation for 6 days of the week and supplied for the 7 th day. Study staff will reinforce the importance of adherence to the treatment schedule at each visit. If the patient misses drug doses in the intensive phase (IP) or continuation phase (CP), it will be compensated at the end of the respective stages over next 15 days, so that the patient receives 60 doses of the assigned regimen in the IP and 120 doses in the CP. Treatment supporters and enablers will facilitate retention of the participants in the study. All treatment is ambulatory and delivered by dedicated DOT provider. We will assess treatment adherence through reviews of treatment card throughout the treatment phase. In case of drug supply for any reason, empty pill cover count will determine adherence. Treatment adherence will be enhanced by reimbursing the transport costs and loss of wages incurred by the participant during the study visits. During the study period, participants will receive food supplement every month and meals during their extended study visits.

\section{Concomitant medication while in the trial}

Concomitant antibiotic treatments of any kind are discouraged during the study period. If required, their usage is restricted for a short course ( $<2$ weeks) period. Concomitant non-antibiotic drug usage is allowed in small quantity. Only those considered necessary for the subject's welfare and are unlikely to interfere with the study medication may be given at the discretion of the investigator. Below are the drugs used to treat $M$. tuberculosis infections which should not be used during this trial: streptomycin, thiacetazone, PAS, dapsone, amoxicillin-clavulanic acid/ clavulanate, clofazimine, capreomycin, any oxazolidinone antibiotic (eg, linezolid), ofloxacin, levofloxacin or moxifloxacin.

\section{Criteria for discontinuation/withdrawal of study participants}

Withdrawal of study participants can occur for any of the following reasons: pregnancy, if the investigators feel that staying in the study is harmful to the patient, if the patient does not follow study procedures or is not available for appointments, if the study sponsor or NIRT or representative of the Drugs Controller General of India decides to stop or cancel the study, if the Data and Safety Monitoring Board recommends halting the study or if the patient wishes to withdraw for any reasons. In such cases, appropriate treatment as per the standard of care treatment available then will be ensured for patients taking into consideration the drug susceptibility pattern of the individual.

\section{Study outcomes}

The primary outcome is the time to sputum culture conversion, which is assessed by the time interval between the date of treatment initiation and the date of acquisition of the first of at least two consecutive negative cultures taken at least 8 days apart. We will also assess, on a weekly basis, the time to positivity, a change in M. tuberculosis $\log _{10}$ colony forming units (CFU) in culture and the proportion of participants with sputum culture negativity in the two treatment arms.

The PK, the area under the concentration curve (AUC), will be assessed through blood sampling on a single day during the first month of ATT, after a minimum of seven doses of RMP and MET (1000 mg). We will estimate the minimum inhibitory concentration (MIC) of each participant's pretreatment infecting isolate from early morning and overnight sputum samples collected at the pretreatment visit. We will also compare the genomics results of metformin and rifampicin with the plasma concentration of these drugs, bacteriological and clinical endpoints along with the drug-drug interaction of metformin and rifampicin.

For the occurrence of treatment-emergent adverse events (TEAEs), clinical investigators will grade clinical and laboratory abnormalities according to the modified Adult Toxicity Table for the Division of Microbiology and Infectious Diseases, National Institutes of Health. ${ }^{10}$ The safety and tolerability analysis will include all patients who were randomised to and received at least one dose of the study regimen.

\section{Participant timeline}

The trial will consist of three stages: screening and enrolment (a maximum of 1 week); the intensive phase of treatment with or without test drug ( 2 months) followed by a continuation phase of standard drugs (4 months). Table 3 shows the schedule of enrolment, interventions and assessments of participants in the trial.

\section{Patient and public involvement}

Patients were not involved in the development of the research question or the design of this study as the scientific problem is still not proven and only in the research arena. However, considering the relevance of the study outcome to public health and policy-makers, the study is discussed in our Institutional Scientific advisory committee, Institutional Ethics committee and Community Advisory Board consisting of a representative from affected community, peers and responsible members of the society. Patients will be involved in the recruitment and conduct of the study. We will disseminate the results of the study widely through meetings, workshops, conference presentation and publication.

\section{Sample size assumption}

Published literature has shown a median time to sputum culture conversion by a liquid medium with daily ATT to be 32 days. ${ }^{11}$ With the addition of metformin, we assumed a $30 \%$ reduction in the time to culture conversion, that is, to approximately 22 days. We estimated that we would require 150 new sputum smear-positive patients to show 
this difference at $80 \%$ power, an alpha level of 0.05 and an HR of 1.5. With the assumption that $5 \%$ of patients will be lost to follow-up or not assessable in the primary analysis, a total target sample size of 158 patients in each treatment arm will be recruited, totalling to 316 patients for the study.

\section{Randomisation procedures}

Permuted block randomization will be done centrally using a computer-generated list of random numbers, stratified by presence or absence of cavities on chest $\mathrm{X}$-ray and highest sputum smear grading $(<2$ or $>2)$. The randomisation sequence will not be available to those who enrol participants. Allocation codes will be generated at the central site, NIRT, and will take place at the time of patient's admission to the study. On receiving an electronic mail request from the study sites, for allocation of a participant to the study, the NIRT statistician will do allocation procedure centrally based on appropriate stratification factors. He/She will then inform the site physician, through e-mail, the study regimen along with the unique study ID for patient enrolment to the study.

\section{Data collection, management and interim analysis}

Study randomisation will be done centrally by the Central Data management (CDM) unit at NIRT, Chennai over email. During the study conduct, data will be collected at sites at baseline and follow-up on predefined case record forms (CRF) and transmitted electronically to the CDM unit. Data correctness and completeness will be checked before sending. The central team will also conduct a periodic quality check of the data. Data collected will include (1) improvement in disease status regarding abetment of symptoms, signs and sputum cultures conversion and (2) safety and tolerability of the regimen concerning both clinical and laboratory adverse events (AEs).

During the trial, all essential trial documents including the source documents, informed consent forms, etc will be stored securely under lock and key at the recruiting sites under the supervision of the site investigator. All e-data will be password protected with limited access to the investigator and their teams alone. The study team will retain the records for a minimum period of 5 years after completion of the study.

The study has two interim analyses planned, viz. after $33 \%$ and $66 \%$ of the enrolled patients have completed 8 weeks of metformin treatment and with sputum culture results available for review. The study also has an additional interim analysis for reported serious adverse events (SAEs) with frequency higher than anticipated. The final analysis will include all enrolled patients when they have completed 6 months of ATT.

\section{Study outcome analysis}

The primary efficacy analysis will compare the median time to culture negativity. This analysis will happen when the last enrolled patient has completed 8 weeks of treatment and will be done using culture results from liquid culture (MGIT) and in those who are not isoniazid or RMP mono-resistant at baseline. Both a modified intent to treat (MITT) and a per protocol (PP) analysis will be conducted. Secondary outcome parameters will include proportion and time to sputum culture positivity using the MGIT system. The safety and tolerability analysis will consist of all patients who were randomised to and received at least one dose of the drug. Based on plasma drug concentrations obtained at different time points, we will calculate certain pharmacokinetic variables related to study drugs. Drug peak concentrations (Cmax) and exposure (AUC) will be linked to time to sputum conversion and occurrence of AEs.

\section{Data and Safety Monitoring Board}

The Data and Safety Monitoring Board (DSMB) for this study comprises TB clinicians, pharmacokinetic specialists and an independent biostatistician. They will review data from this trial on a regular basis, including incidence progress of the trial, and detect evidence of early safety issues for the trial participants with a specific focus on grade 3 or 4 AEs, SAEs and treatment discontinuations due to AE. Based on the results, DSMB shall make recommendations on continuing or terminating/modifications to the trial.

\section{Clinical site monitoring and quality assurance}

An independent study monitor, appointed by the sponsor, will be responsible for monitoring data quality by trial standard operating procedures. Based on the monitoring plan, field visit and audit will be performed at different stages. All participant records, CRFs and other source documents for the patients recruited in this study will be made available for review by the monitors. A meeting of the investigators of each local site will convene monthly via web-based remote conference system to share the progress of the study and discuss with the problems met during the trial conduct. During the conduct of the research, any critical protocol modifications will be informed to the IEC, trial registry and, if relevant, to the trial participants.

\section{Confidentially of trial data}

The processing of personal data in this trial will be limited to those data that are reasonably necessary to investigate the antibacterial activity, safety and tolerability of the investigational product used in this trial. These data will be processed with adequate precautions to ensure confidentiality. Trained professional will collect al study data with the utmost sensitivity and confidentiality. The study participant will be informed during the informed consent process that: the monitor(s) the IEC, and the regulatory authorities will be granted direct access to the participant's original medical records for verification of clinical trial procedures and/or data, without violating the confidentiality of the participant, to the extent permitted by the applicable laws. Otherwise, only the study investigator and his/her team will have access to the trial data. 


\section{ETHICS AND DISSEMINATION}

This trial will proceed as per the current ICH Good Clinical Practice and the ICMR ethical guidelines for biomedical research in human participants. Compliance with this standard provides public assurance that the rights, safety and well-being of trial subjects are protected, consistent with the principles that have their origin in the Declaration of Helsinki, and that the clinical trial data are credible. The confidentiality of the study participants will be protected throughout the study period as per provision in the Indian-GCP and applicable regulations by laws of India. The processing of personal data in this trial will be limited to those data that are reasonably necessary to investigate the antibacterial activity, safety and tolerability of the investigational product used in this trial.

The study principal investigator holds primary responsibility for the preparation of manuscripts and materials for result dissemination and publication. Once the trial is complete, the investigators anticipate publishing results of this study in several peer-reviewed scientific journals, present the abstracts in meetings, to stakeholders and policymakers and share the results widely with the programme managers. During all these processes, details of the trial participant will remain confidential.

\section{DISCUSSION}

Currently, for the management of patients with newly diagnosed sputum smear-positive drug-sensitive pulmonary $\mathrm{TB}, 6$-month regimens are used in more than 90 countries that are evaluated to be effective. ${ }^{12}$ Newer anti-TB drugs are also in the pipeline. However, all types of treatment currently available are pathogen-targeted and with toxic drugs. Drug toxicity can lead to poor treatment adherence resulting in treatment failure and development of resistance. As the pathogen-targeted strategies may lead to the development of acquired microbial resistance, new 'host-targeted' adjunct therapeutic approaches not only are less likely to engender microbial resistance but also augment protective host immune responses, thus accelerating bacterial clearance from the system.

Metformin has an inhibitory effect on mitochondrial complex I, inhibition of which has been found to increase the AMP/ATP ratio. ${ }^{13} 14$ The altered cellular energy status induces activation of AMPK, a serine/threonine kinase, and acts as an energy sensor. ${ }^{15}$ Activation of AMPK by metformin stimulates endothelial nitric oxide synthase activity which leads to bacterial killing. ${ }^{16}$ Metformin also acts through AMPK-independent mechanisms. It promotes phagocytosis, phagolysosome fusion and autophagy in macrophages. Macrophages exposed to metformin had higher bactericidal capacity attributed to increased mitochondrial reactive oxidative species (ROS) production required for bacterial killing. ${ }^{17}$ Approximately $90 \%$ of the newly diagnosed sputum-positive patients are sensitive to isoniazid $(\mathrm{H})$ and rifampicin $(\mathrm{R})$, adding the drug metformin would have a beneficial effect in the early killing of intracellular bacteria by influencing the host immunity. Experiments in mice treated with MET $(200 \mathrm{mg} / \mathrm{kg}$ ) along with $\mathrm{H} 10 \mathrm{mg} / \mathrm{kg}$ showed not only a considerable reduction in the bacillary load in the lungs but also reduced areas of lung tissue damage compared with $\mathrm{H}$-alone treatment. ${ }^{9}$ This dose of MET is equivalent to approximately $1200 \mathrm{mg}$ / day for a $60 \mathrm{~kg}$ human and this dose of MET will be used in this trial along with $\mathrm{H}, \mathrm{R}$, $\mathrm{E}$ and $\mathrm{Z}$ in the intensive phase of daily ATT. High dose of metformin has also been used in clinical practice for the management of diabetic individuals with and without TB and in non-diabetic conditions like polycystic ovarian syndrome and obesity. ${ }^{18} 19$ Though WHO recommends 2HRZE/4-hour regimen for new patients with pulmonary $\mathrm{TB}$, given the high prevalence of isoniazid resistance in the country ( $11 \%$ in new sputum-positive patients), the Revised National TB control programme of India recommends using three drugs in the continuation phase, that is, 2HRZE/4HRE regimen which will be followed in this trial. $^{20}$

We are proposing a phase IIB trial looking at sputum-culture conversion to negative over a 2-month period as studies have shown a correlation between positive 2-month sputum culture status and subsequent relapse. However, limitations of this design include the binary outcome of 2-month sputum culture endpoint, hence requiring larger samples size to prove the benefit. To overcome this limitation, we plan serial sputum colony counting (SSCC) and time-to-detection (TTD). SSCC involves counting of viable $M$. tuberculosis bacilli at various time points during the 8-week period. This measurement will demonstrate the rapid bacillary killing in the early phase and track the rate and pattern of culture conversion throughout the 8 weeks, thereby providing a better marker for culture conversion. ${ }^{21}$ However, TTD in liquid culture is considered an even better choice than SSCC as the complications of counting viable colonies is replaced by automated measurement of detection time of the bacilli throughout the 8-week period. In our study, as the outcome measures of interest (primarily bacteriological) are based on objective microbiology, the bacteriology laboratory staff will be blinded to the patient's treatment. Safety assessments will be defined as objectively as possible, using predefined grading criteria for laboratory abnormalities and AEs. The pharmacokinetic aspect of the study is based on a population approach which is facilitated by the intensive-sparse sampling design. The study will estimate the pharmacokinetic exposure (AUC0-24) for all study participants. Several alternative biomarkers of treatment response are also being evaluated using these samples.

Results from METRIF study will complement observations from other retrospective and case-control studies that showed metformin to be a protective agent against TB infection among people with diabetes. ${ }^{22}{ }^{23}$ As studies did not show any dose-dependent protection in metformin users for TB, we will be using $500 \mathrm{mg}$ dose for the first week followed by $1000 \mathrm{mg}$ for the remaining 7 weeks. This dose escalation is being done to reduce the gastrointestinal side 
effects of metformin. If the study regimen is successful, it will pave the way to evaluate shorter regiments for the treatment of pulmonary TB. This trial will establish a new standard of care for DS-pulmonary TB that will not only reduce the number of required clinic visits by the patients but also decrease the proportion of patients who fail to complete the full course of therapy. It should also reduce the overall burden on the healthcare system. If the study regimens are shown to be superior or at least non-inferior to the control regimen, then the procedure represents an even greater advantage for both patients and TB control programmes throughout the world.

\section{Author affiliations}

${ }^{1}$ ICMR-National Institute for Research in Tuberculosis, Chennai, India

${ }^{2}$ All India Institute of Medical Sciences, New Delhi, India

${ }^{3}$ Open Source Pharma Foundation, Chennai, India

${ }^{4}$ Indian Council of Medical Research-India TB Research Consortium, New Delhi, India

${ }^{5}$ World Health Organisation, Geneva, Switzerland

Contributors CP and SS: conceived and designed the study. CP, PKB, MN, CPr, HK, NSG, RG, SJ, TB, SS: development and writing of the study protocol and this manuscript.

Funding India TB Research Consortium, Indian Council of Medical Research, New Delhi and Open Source Pharma Foundation, Bangalore, India provided funding for the study. Trial Sponsor: Indian Council of Medical Research - National Institute for Research in Tuberculosis.

Competing interests None declared.

Patient consent for publication Not required.

Provenance and peer review Not commissioned; externally peer reviewed.

Open access This is an open access article distributed in accordance with the Creative Commons Attribution Non Commercial (CC BY-NC 4.0) license, which permits others to distribute, remix, adapt, build upon this work non-commercially, and license their derivative works on different terms, provided the original work is properly cited, appropriate credit is given, any changes made indicated, and the use is non-commercial. See: http://creativecommons.org/licenses/by-nc/4.0/.

\section{REFERENCES}

1. World Health Organization. Global Tuberculosis Report. Geneva, 2017. Accessed. http://www.who.int/tb/publications/global_report/ en/

2. Kuijl C, Savage ND, Marsman M, et al. Intracellular bacterial growth is controlled by a kinase network around PKB/AKT1. Nature 2007;450:725-30.

3. Ejim L, Farha MA, Falconer SB, et al. Combinations of antibiotics and nonantibiotic drugs enhance antimicrobial efficacy. Nat Chem Biol 2011;7:348-50.
4. Bhatt $K$, Salgame P. Host innate immune response to Mycobacterium tuberculosis. J Clin Immunol 2007;27:347-62.

5. Behar SM, Divangahi M, Remold HG. Evasion of innate immunity by Mycobacterium tuberculosis: is death an exit strategy? Nat Rev Microbiol 2010;8:668-74.

6. Gutierrez MG, Master SS, Singh SB, et al. Autophagy is a defense mechanism inhibiting BCG and Mycobacterium tuberculosis survival in infected macrophages. Cell 2004;119:753-66.

7. Kim J, Kundu M, Viollet B, et al. AMPK and mTOR regulate autophagy through direct phosphorylation of Ulk1. Nat Cell Biol 2011;13:132-41.

8. Kumar D, Nath L, Kamal MA, et al. Genome-wide analysis of the host intracellular network that regulates survival of Mycobacterium tuberculosis. Cell 2010;140:731-43.

9. Singhal A, Jie L, Kumar P, et al. Metformin as adjunct antituberculosis therapy. Sci Trans/ Med 2014;6:263ra159.

10 . Division of AIDS (DAIDS) Table for Grading the Severity of Adult and Pediatric Adverse Events. Version 2.1, 2017. (Accessed on 29 Mar 2018). https://rsc.tech-res.com/docs/default-source/safety/daids-aegrading-table-mar2017.pdf

11. Wang JY, Wang JT, Tsai TH, et al. Adding moxifloxacin is associated with a shorter time to culture conversion in pulmonary tuberculosis. Int J Tuberc Lung Dis 2010;14:65-71.

12. Fox W, Ellard GA, Mitchison DA. Studies on the treatment of tuberculosis undertaken by the British Medical Research Council tuberculosis units, 1946-1986, with relevant subsequent publications. Int J Tuberc Lung Dis 1999;3:S231-79.

13. El-Mir MY, Nogueira V, Fontaine E, et al. Dimethylbiguanide inhibits cell respiration via an indirect effect targeted on the respiratory chain complex I. J Biol Chem 2000;275:223-8.

14. Owen MR, Doran E, Halestrap AP. Evidence that metformin exerts its anti-diabetic effects through inhibition of complex 1 of the mitochondrial respiratory chain. Biochem J 2000;348 Pt 3:607-14.

15. Hardie DG. The AMP-activated protein kinase pathway--new players upstream and downstream. J Cell Sci 2004;117:5479-87.

16. Davis BJ, Xie Z, Viollet B, et al. Activation of the AMP-activated kinase by antidiabetes drug metformin stimulates nitric oxide synthesis in vivo by promoting the association of heat shock protein 90 and endothelial nitric oxide synthase. Diabetes 2006;55:496-505.

17. Tan HY, Wang N, Li S, et al. The reactive oxygen species in macrophage polarization: Reflecting its dual role in progression and treatment of human diseases. Oxid Med Cell Longev 2016;2016:1-16.

18. Lord JM, Flight IH, Norman RJ. Metformin in polycystic ovary syndrome: systematic review and meta-analysis. BMJ 2003;327:951.

19. Levri KM, Slaymaker E, Last A, et al. Metformin as a treatment for overweight and obese adults. Ann Fam Med 2005;3:457-61.

20. Technical and operational guidelines for TB control in India. 2016. https://tbcindia.gov.in/showfile.php?lid=3219 (Accessed on $17 \mathrm{Nov}$ 2018).

21. Bark CM, Furin JJ, Johnson JL. Approaches to clinical trials of new anti-TB drugs. Clin Investig 2012;2:359-70.

22. Sullivan T, Ben Amor Y, Amor BY. The co-management of tuberculosis and diabetes: challenges and opportunities in the developing world. PLoS Med 2012;9:e1001269.

23. Marupuru S, Senapati P, Pathadka S, et al. Protective effect of metformin against tuberculosis infections in diabetic patients: an observational study of south Indian tertiary healthcare facility. Braz $J$ Infect Dis 2017;21:312-6. 